\title{
A relation between the Lorentzian inversion and the Bateman transformation
}

\author{
Katsunori ShimomurA*
}

\begin{abstract}
In our previous paper [1], we proved that every transformation which preserves the wave equation is a similarity or a Lorentzian inversion composed with similarities or a Bateman transformation composed with similarities. In this paper, we give several relations between Bateman transformation and Lorentzian inversion. We also prove that only Lorentzian inversion or Bateman transformation is enough to generate the set of all transformations which preserve the wave equation.
\end{abstract}

\section{Introduction and known results}

In [1], we considered Liouville type theorem associate with the wave equation, and proved that every conformal mapping for the Lorentzian metric is one of the following: a similarity, a inversion composed with similarities, a Bateman transformation composed with similarities. We also determined the transformations which preserve the solutions of the wave equation. The purpose of this note is to discuss relations among these transformations and to give generators of the group of all transformations which preserve the wave equation (see Theorems 3.1 and 3.2 below).

Let $\mathbb{R}^{n+1}$ be the $(n+1)$-dimensional Euclidean space $(n \geqq 2)$, and denote its point by $x=\left(x_{0}, x_{1}, \ldots, x_{n}\right)$. We introduce Lorentzian scalar product $\langle\cdot, \cdot\rangle$ on $\mathbb{R}^{n+1}$ defined by

$$
\langle x, y\rangle=-x_{0} y_{0}+x_{1} y_{1}+\cdots+x_{n} y_{n} .
$$

The vector space $\mathbb{R}^{n+1}$ with this Lorentzian scalar product is called Lorentzian $(n+1)$ space, and is denoted by $\mathbb{R}^{1, n}$.

Let $D, E \subset \mathbb{R}^{1, n}$ be domains and $f: D \rightarrow E$ a $C^{1}$-mapping. The first derivative $f^{\prime}(x)$ of $f$ at $x \in D$ is a linear mapping $\mathbb{R}^{1, n} \rightarrow \mathbb{R}^{1, n}$ such that

$$
f^{\prime}(x) u=\lim _{h \rightarrow 0} \frac{f(x+h u)-f(x)}{h}, \quad u \in \mathbb{R}^{1, n} .
$$

Received 20 June 2011; revised 13 July 2011

2000 Mathematics Subject Classification. 31B99, 35K99, 35A30

Key Words and Phrases. transformation preserves wave equation, Lorentzian inversion, Bateman transformation

*Partially supported by Grant-in-aid for Scientific Research (C) No.22540169, Japan Society for the Promotion of Science.

*Ibaraki University, Mito, Ibaraki, 310-8512, Japan.(shimomur@mx.ibaraki.ac.jp) 
Definition 1.1. A $C^{1}$-mapping $f: D \rightarrow E$ is said to be Lorentzian conformal if there exists a function $\lambda(x)=\lambda_{f}(x)>0$ defined on $D$ such that

$$
\left\langle f^{\prime}(x) u, f^{\prime}(x) v\right\rangle=\lambda(x)^{2}\langle u, v\rangle
$$

holds for all $x \in D$ and all $u, v \in \mathbb{R}^{1, n}$.

Note that a composition of two Lorentzian conformal mapping is also Lorentzian conformal.

Next, we consider the wave equation

$$
W u:=\frac{\partial^{2} u}{\partial x_{0}^{2}}-\sum_{j=1}^{n} \frac{\partial^{2} u}{\partial x_{j}^{2}}=0
$$

on $\mathbb{R}^{1, n}$ and transformations which preserve the solutions of the wave equation.

Definition 1.2. Let $D \subset \mathbb{R}^{n+1}$ be a domain, $f: D \rightarrow \mathbb{R}^{1, n}$ a $C^{2}$-mapping, and $\varphi$ a positive $C^{2}$-function on $D$. A pair $(f, \varphi)$ is called a transformation which preserves the wave equation, if $f$ and $\varphi$ satisfy the following conditions:

(1) $f^{\prime}(x)$ is non-degenerate for all $x \in D$.

(2) For every solution $u$ of the wave equation on $\mathbb{R}^{1, n}, \varphi \cdot(u \circ f)$ satisfies the wave equation on $D$.

Note that if $\left(f, \varphi_{f}\right)$ and $\left(g, \varphi_{g}\right)$ are transformations which preserve the wave equation such that the image of $f$ is contained in the domain of $g$, then the composition $\left(g \circ f,\left(\varphi_{g} \circ f\right) \cdot \varphi_{f}\right)$ is also a transformation which preserves the wave equation.

Let

$$
\mathrm{O}(1, n):=\left\{R \in \mathrm{GL}(n+1, \mathbb{R}) ;\langle R x, R y\rangle=\langle x, y\rangle \text { for all } x, y \in \mathbb{R}^{1, n}\right\} .
$$

In the following, we list fundamental Lorentzian conformal mappings and corresponding transformations which preserve the wave equation.

Example 1.1 (Lorentzian similarity). The mapping $f(x)=\alpha R x+a$, where $\alpha \in \mathbb{R}$, $\alpha>0, R \in \mathrm{O}(1, n)$, and $a \in \mathbb{R}^{1, n}$, is a Lorentzian conformal mapping defined on $\mathbb{R}^{1, n}$. The pair $(f, 1)$ is a transformation which preserves the wave equation.

Example 1.2 (Lorentzian inversion). The mapping $K(x)=\frac{x}{\langle x, x\rangle}$ is a Lorentzian conformal mapping defined on each component of $\left\{x \in \mathbb{R}^{1, n} ;\langle x, x\rangle \neq 0\right\}$. $K$ satisfies $K^{-1}=K$. The pair $\left(K,|\langle x, x\rangle|^{\frac{1-n}{2}}\right)$ is a transformation which preserves the wave equation.

Example 1.3 (Bateman transformation). The mapping

$$
B(x)=\left(\frac{\langle x, x\rangle+1}{2\left(x_{0}+x_{1}\right)}, \frac{\langle x, x\rangle-1}{2\left(x_{0}+x_{1}\right)}, \frac{x_{2}}{x_{1}+x_{0}}, \ldots, \frac{x_{n}}{x_{1}+x_{0}}\right)
$$

is a Lorentzian conformal mapping defined on each component of $\left\{x \in \mathbb{R}^{1, n} ; x_{0}+x_{1} \neq\right.$ $0\}$. The pair $\left(B,\left|x_{0}+x_{1}\right|^{\frac{1-n}{2}}\right)$ is a transformation which preserves the wave equation. 
In [1], we proved the following two theorems:

Theorem A. Let $n \geqq 2$ and $f$ be a Lorentzian conformal $C^{4}$-mapping defined on a domain $D \subset \mathbb{R}^{1, n}$. Then $f$ has one of the following forms:

$$
\begin{aligned}
& f(x)=\alpha R K(x-a)+b, \\
& f(x)=\alpha R B(S(x-a))+b, \\
& f(x)=\alpha R x+b,
\end{aligned}
$$

where $\alpha>0, R, S \in \mathrm{O}(1, n), a, b \in \mathbb{R}^{1, n}$.

Theorem B. Let $n \geqq 2, f$ be a $C^{4}$-mapping, and $\varphi$ a positive function defined on a domain $D \subset \mathbb{R}^{1, n}$. If $(f, \varphi)$ be a transformation which preserve the wave equation, then $f$ and $\varphi$ have one of the following forms:

$$
\begin{array}{llrl}
f(x) & =\alpha R K(x-a)+b, & \varphi(x) & =C|\langle x-a, x-a\rangle|^{\frac{1-n}{2}}, \\
f(x) & =\alpha R B(S(x-a))+b, & \varphi(x) & =C|\langle v, S(x-a)\rangle|^{n-1}, \\
f(x) & =\alpha R x+b, & \varphi(x) & =C,
\end{array}
$$

where $\alpha>0, R, S \in \mathrm{O}(1, n), a, b \in \mathbb{R}^{1, n}$, and $v=(-1,1,0, \ldots, 0) \in \mathbb{R}^{1, n}$.

\section{Relations between Bateman transformation and Lorentzian inversion}

In the last section, we introduced Lorentzian scalar product. Let $J$ be the $(n+$ 1) $\times(n+1)$ diagonal matrix

$$
J=\operatorname{diag}(-1,1, \ldots, 1) .
$$

Then the Lorentzian scalar product $\langle\cdot, \cdot\rangle$ is written by the Euclidean scalar product $(\cdot, \cdot)$ as

$$
\langle x, y\rangle=(J x, y)=(x, J y), \quad x, y \in \mathbb{R}^{1, n} .
$$

Recall that $\mathrm{O}(1, n)=\left\{R ;{ }^{t} R J R=J\right\}=\left\{R ; R J^{t} R=J\right\} . \mathrm{O}(1, n)$ is the group of all matrices which preserve the Lorentzian scalar product.

For $a \in \mathbb{R}^{1, n}$, we define the translation operator $T_{a}$ by $T_{a}(x)=x-a$. Clearly $T_{a}(A x)=A T_{A^{-1} a}(x)$ holds for any $A \in \mathrm{GL}(n+1, \mathbb{R})$.

Proposition 2.1. Let $u \in \mathbb{R}^{1, n}$ such that $\langle u, u\rangle \neq 0$. Then

$$
K T_{u} K=\frac{1}{\langle u, u\rangle}\left(T_{u} S_{u} K T_{\hat{u}}\right)
$$

where $\hat{u}=\frac{u}{\langle u, u\rangle}=K(u)$ and $S_{u}(x)=x-2 \frac{\langle x, u\rangle}{\langle u, u\rangle} u$.

Proof. First,

$$
\left(K T_{u} K\right)(x)=\frac{\langle x, x\rangle^{-1} x-u}{\left\langle\langle x, x\rangle^{-1} x-u,\langle x, x\rangle^{-1} x-u\right\rangle}=\frac{x-\langle x, x\rangle u}{1-2\langle x, u\rangle+\langle x, x\rangle\langle u, u\rangle} .
$$


Since $\langle u, u\rangle \neq 0$ and $\langle\hat{u}, \hat{u}\rangle=\frac{1}{\langle u, u\rangle}$, the right hand side equals to

$$
\begin{gathered}
\frac{x-\langle x, x\rangle u}{\langle u, u\rangle(\langle\hat{u}, \hat{u}\rangle-2\langle x, \hat{u}\rangle+\langle x, x\rangle)}=\frac{1}{\langle u, u\rangle}\left(\frac{x-\hat{u}-2\langle x-\hat{u}, \hat{u}\rangle u}{\langle x-\hat{u}, x-\hat{u}\rangle}-u\right) \\
=\frac{1}{\langle u, u\rangle} T_{u}\left(K\left(T_{\hat{u}} x\right)-2 \frac{\left\langle K\left(T_{\hat{u}} x\right), u\right\rangle}{\langle u, u\rangle} u\right)=\frac{1}{\langle u, u\rangle}\left(T_{u} S_{u} K T_{\hat{u}}\right)(x) .
\end{gathered}
$$

We remark that $S_{u} \in \mathrm{O}(1, n)$.

Let $u \in \mathbb{R}^{1, n}$ satisfy $u \neq 0$ and $\langle u, u\rangle=0$. Then $\langle u, J u\rangle=(u, u) \neq 0$. We define the mapping $B_{u}$ on $\{x ;\langle x, u\rangle \neq 0\}$ by

$$
B_{u}(x)=\frac{1}{\langle x, u\rangle}\left(\frac{\langle x, x\rangle}{2} u-\frac{1}{\langle u, J u\rangle} J u+x-\frac{\langle x, J u\rangle}{\langle u, J u\rangle} u-\frac{\langle x, u\rangle}{\langle u, J u\rangle} J u\right) .
$$

Put $u_{0}=(1,1,0, \ldots, 0)$. Then the Bateman mapping $B$ can be obtained by $B_{u_{0}}$ as

$$
B=B_{u_{0}} J=J B_{J u_{0}} .
$$

Proposition 2.2. Let $u \in \mathbb{R}^{1, n}$ satisfy $u \neq 0$ and $\langle u, u\rangle=0$. Then $B_{u}$ is Lorentzian conformal. We also have $B_{u}^{-1}=B_{-u}$ and

$$
B_{u}=T_{u^{*}} K T_{-\frac{1}{2} u} K T_{u^{*}},
$$

where $u^{*}=\frac{J u}{\langle u, J u\rangle}$.

Proof. We first prove (2.2). Since $\langle u, u\rangle=0$ and $\left\langle u, u^{*}\right\rangle=1$,

$$
\left(K T_{-\frac{1}{2} u} K\right)(x)=\frac{x+\frac{1}{2}\langle x, x\rangle u}{1+\langle x, u\rangle+\frac{1}{4}\langle x, x\rangle\langle u, u\rangle}=\frac{x+\frac{1}{2}\langle x, x\rangle u}{1+\langle x, u\rangle}=\frac{x+\frac{1}{2}\langle x, x\rangle u}{\left\langle u^{*}, u\right\rangle+\langle x, u\rangle} .
$$

Note that $\left\langle u^{*}, u^{*}\right\rangle=0$. Putting $y=x+u^{*}=T_{u^{*}}^{-1}(x)$, the right hand side equals to

$$
\begin{aligned}
& \frac{y-u^{*}+\frac{1}{2}\left\langle y-u^{*}, y-u^{*}\right\rangle u}{\langle y, u\rangle}=\frac{1}{\langle y, u\rangle}\left(y-\frac{J u}{\langle u, J u\rangle}+\frac{\langle y, y\rangle}{2} u-\left\langle y, \frac{J u}{\langle u, J u\rangle}\right\rangle u\right) \\
& =\frac{1}{\langle y, u\rangle}\left(\frac{\langle y, y\rangle}{2} u-\frac{J u}{\langle u, J u\rangle}+y-\frac{\langle y, J u\rangle}{\langle u, J u\rangle} u-\frac{\langle y, u\rangle}{\langle u, J u\rangle} J u\right)+\frac{J u}{\langle u, J u\rangle} \\
& =B_{u}(y)+u^{*}=\left(T_{u^{*}}^{-1} B_{u} T_{u^{*}}^{-1}\right)(x) .
\end{aligned}
$$

Therefore $B_{u}=T_{u^{*}} K T_{-\frac{1}{2} u} K T_{u^{*}}$ holds.

Next, the conformality of $B_{u}$ follows immediately from this equation.

Finally, to show $B_{u}^{-1}=B_{-u}$, note that $(-u)^{*}=-u^{*}$ holds by definition. Then

$$
B_{u}^{-1}=T_{-u^{*}} K^{-1} T_{\frac{1}{2} u} K^{-1} T_{-u^{*}}=T_{(-u)^{*}} K T_{-\frac{1}{2}(-u)} K T_{(-u)^{*}}=B_{-u}
$$

because $K^{-1}=K$. 
Proposition 2.3. We have the following relation:

$$
B^{2}=J_{1} K=K J_{1}
$$

where $J_{1}=\operatorname{diag}(1,-1,1, \ldots, 1) \in \mathrm{O}(1, n)$.

Proof. Put $y=B(x)$. Then

$$
\frac{1}{y_{0}+y_{1}}=\frac{x_{0}+x_{1}}{\langle x, x\rangle},\langle y, y\rangle=\frac{x_{0}-x_{1}}{x_{0}+x_{1}}, \frac{\langle y, y\rangle+1}{2}=\frac{x_{0}}{x_{0}+x_{1}}, \frac{\langle y, y\rangle-1}{2}=\frac{-x_{1}}{x_{0}+x_{1}} .
$$

Hence

$$
\begin{aligned}
B^{2}(x) & =B(y)=\frac{1}{y_{0}+y_{1}}\left(\frac{\langle y, y\rangle+1}{2}, \frac{\langle y, y\rangle-1}{2}, y_{2}, \ldots, y_{n}\right) \\
& =\left(\frac{x_{0}}{\langle x, x\rangle}, \frac{-x_{1}}{\langle x, x\rangle}, \frac{x_{2}}{\langle x, x\rangle}, \ldots, \frac{x_{n}}{\langle x, x\rangle}\right)=\left(J_{1} K\right)(x)=\left(K J_{1}\right)(x) .
\end{aligned}
$$

\section{Results}

In this way, we obtain the following theorems from Theorems A and B and relations (2.1), (2.2), and (2.3).

Theorem 3.1. $\quad$ Let $n \geqq 2$. Then the set of all Lorentzian conformal $C^{4}$-mappings can be generated by $K$ and Lorentzian similarities. And the set of all $C^{4}$-transformations which preserve the wave equation can be generated by Lorentzian inversion $K$ and Lorentzian similarities.

Theorem 3.2. Let $n \geqq 2$. Then the set of all Lorentzian conformal $C^{4}$-mapping can be generated by $B$ and Lorentzian similarities. And the set of all $C^{4}$-transformations which preserve the wave equation can be generated by Bateman transformation $B$ and Lorentzian similarities.

Acknowledgements: The author would like to express his gratitude to Professor M. Nishio for his valuable suggestions and comments.

\section{References}

[1] K. Shimomura, Liouville type theorem associated with the wave equation, Math. J. Ibaraki Univ., 43 (2011), 51-64. 